\section{MS35 O1}

The use of X-ray diffraction for process optimization in the minerals and metallurgical industry Johan de $\underline{\text { Villiers }}{ }^{\mathrm{a}}$, Sabine Verryn ${ }^{\mathrm{b}}{ }^{a}$ Department of $\overline{\text { Materials }}$ Science and Metallurgical Engineering ${ }^{b}$ Department of University of Pretoria, Pretoria, 0002 South Africa

E-mail: johan.devilliers@up.ac.za

Keywords: Rietveld; metallurgy; mineral science Through the application of the Rietveld method, X-ray diffraction analysis has developed into a fully quantitative method for phase analysis of metallurgical products. The Rietveld method itself has progressed from an unstable method that required constant operator intervention to a stable and robust method suited even for routine analysis. Crystal structure determination still occupies a central position in the proper characterization of metallurgical products. It provides the basic structural information necessary for the determination of mineral stoichiometry and for elucidating solid solution behaviour. An excellent example is the crystal structure analysis of the SFCA phases that form when iron ores are sintered. Another example is the elucidation of the arsenopyrite structure that eluded crystal chemists for years.

The use of unit cell data in the heavy minerals industry is a good example of a case where conventional microchemical analysis is unable to provide an answer to the extent of solid solution behaviour in ilmenite roasting. $\mathrm{XRD}$ is used to determine the amount of roasting necessary for the development of magnetization to be able to separate ilmenite from chromite.

Phase quantification is probably the most widely used application of XRD analysis. It is widely used for materials balance in the heavy minerals and cement industries, and it is finding novel applications in the iron and steel industry. Evaluation of refractories by XRD analysis is also supplementing conventional microscopic analysis.

In the minerals processing industry, XRD analysis is used to identify problematic minerals in ores, such as clays in heavy minerals recovery, the presence of talc in flotation circuits, as well as sulphide minerals causing acid mine drainage.

Applications of XRD in high-temperature metallurgical processes range from the phase chemistry of iron ore sinters, the evaluation of subsolidus reactions in titania slags, and the identification of furnace accretions.

Despite the success of the Rietveld method in phase quantification, the method still needs further development in the quantification of closely related or polytypic phases. The quantification of pyrrhotite phases using various Rietveld programmes still gives ambiguous results. This is also true for the different SFCA phases in iron ore sinters. Clearly the analysis of similar phases, differing only in the presence of weak superlattice reflections is still problematic.

\author{
MS35 O2 \\ Nanomorphology and surface potential of layer \\ silicates by scanning probe microscopy Giovanni Valdre \\ Department of Earth and Geo-Environmental Sciences, \\ University of Bologna, Italy \\ E-mail:giovanni.valdre@unibo.it
}

The knowledge of the surface properties of layer silicates is of great importance to understand both basic and applicative technological issues, such as, for example liquid/surface interactions, microfluidity, friction or tribology and bio-molecules self-assembly and adhesion. Recent developments of Scanning Probe Microscopy (SPM) have widened the spectrum of possible investigations that can be performed at a nanometric level on the surface of minerals. They range from physical properties such as surface potential and electric field topological determination to chemical and spectroscopical analysis both in air, in liquid or in a gaseous environment. After an introduction illustrating the theory and basics of instrumentation which stand behind SPM, we will present its recent achievements in the field of the characterization of nanomorphology and surface potential properties of minerals, in particular the behaviour of some layer silicates. Applications to mineral hydrophilicity/hydrophobicity determination, organic surface interactions of layer silicates, friction and nanotribology of minerals and adhesion issues on applicative basis will be addressed.

SPM was used to assess the force-curve, force-volume and surface potential characteristics of layer silicates by working in Electric Force Microscopy (static and dynamic EFM) and in Kelvin probe modes of operation. For instance, electric force microscopy allowed us to measure the thickness of silicate layers, to experimentally verify the layer polarity and, from frequency, amplitude, phase modulation and Kelvin analysis, to derive the electrostatic force experienced by the probe. We can then directly relate these measurements with the electrostatic force gradient at the mineral surface $[1,2]$.

The transverse dynamic force microscopy, also known as shear force microscopy (ShFM) will be introduced and examples on the investigation of attractive, adhesive and shear forces of water onto layer silicate and other mineral surfaces will be presented. The study of water in confined geometries is very important because it can provide simple models for fluid/mineral interactions. Water is confined between a resonating ShFM probe and layer mineral surfaces. The microscope probe was mounted vertically and perpendicularly to the mineral surface and set in horizontal oscillation.

The oscillation amplitude and the corresponding phase signal were quantitatively measured as a function of the confinement distance. When the liquid film thickness was less then a critical length the oscillation amplitude curve presented a step-like trend suggesting that the liquid molecules were organized in layers. By using an harmonic oscillator model it is possible to calculate the variation in the elastic and viscous stress response of the confined liquid and the storage modulus $G^{\prime}$ and the loss modulus G', were determined [3].

[1] Valdre, G.; Moro, D.; Bacchin, P., Proc. XVI Intl. Microsc. Congress, 2007, 2, 766.

[2] Valdre, G., Eur. J. Min. 2007, in press.

[3] Antognozzi, M.; Protti, A.; Miles, M.J.; Valdre, G., GeoActa, 2003, 2, 101 . Keywords: $\begin{aligned} & \text { Layer silicates, } \\ & \text { microscopy, Industrial minerals }\end{aligned}$ 